\title{
Unique research and development for fibres and textiles on an industrial scale
}

\section{Introduction}

The "Technical Fibres" division of the Institut für Textiltechnik of the RWTH Aachen University (ITA) is already developing innovative fibres of tomorrow today. As the world's leading contact for industryoriented research in mono- and multifilament melt spinning, the department is the main source for information on process optimization, digitisation, material development, recycling and functionalization (Figure 1).

The ITA Group's ability to cover the entire textile value chain from natural and synthetic raw materials, through further processing, to the finished end product is unique. As one of the leading international research and training service providers for fibre-based high-performance materials, semi-finished textile products and their manufacturing processes, the ITA Group has a long-lasting history of coorperation with partners from research and industry. In public and bilateral research projects, the development of innovative products and processes is driven forward collaboratively, and strategic consulting on current topics such as recycling and digitisation is provided (Figure 2).

The institute's technical centre is where the textile chain is investigated, beginning with fibre production and proceeding through fibre post-processing with the potential steps such as coating, winding, stretching or twisting. Subsequently, the production of surfaces
Volume 7 Issue 2 - 202I

\author{
Melina Sachtleben, Robert Brüll, Franz \\ Pursche \\ Heads of Department at the Institute of Textile Technology at \\ RWTH Aa-chen University (ITA), USA
}

Correspondence: Melina Sachtleben, MSc, Heads of Department at the Institute of Textile Technology at RWTH Aa-chen University (ITA), USA,

Email Melina.Sachtleben@ita.rwth-aachen.de

Received: January 13, 2021 | Published: April 20, 2021

follows in the form of woven fabrics, knitted fabrics, warp-knitted fabrics, scrims, braids (3D braiding), nonwovens and preforms.

Textile semi-finished products, finished components and smart textiles for a wide range of applications represent the end of the textile value chain represented at ITA. Textile-based luminous concrete, 3D circular knitted fabrics, textile touch pads, sensor-based protective equipment, sound-absorbing home textiles and pest-control functional fibres are just a few examples of the diverse textile developments at the institute (Figure 3).

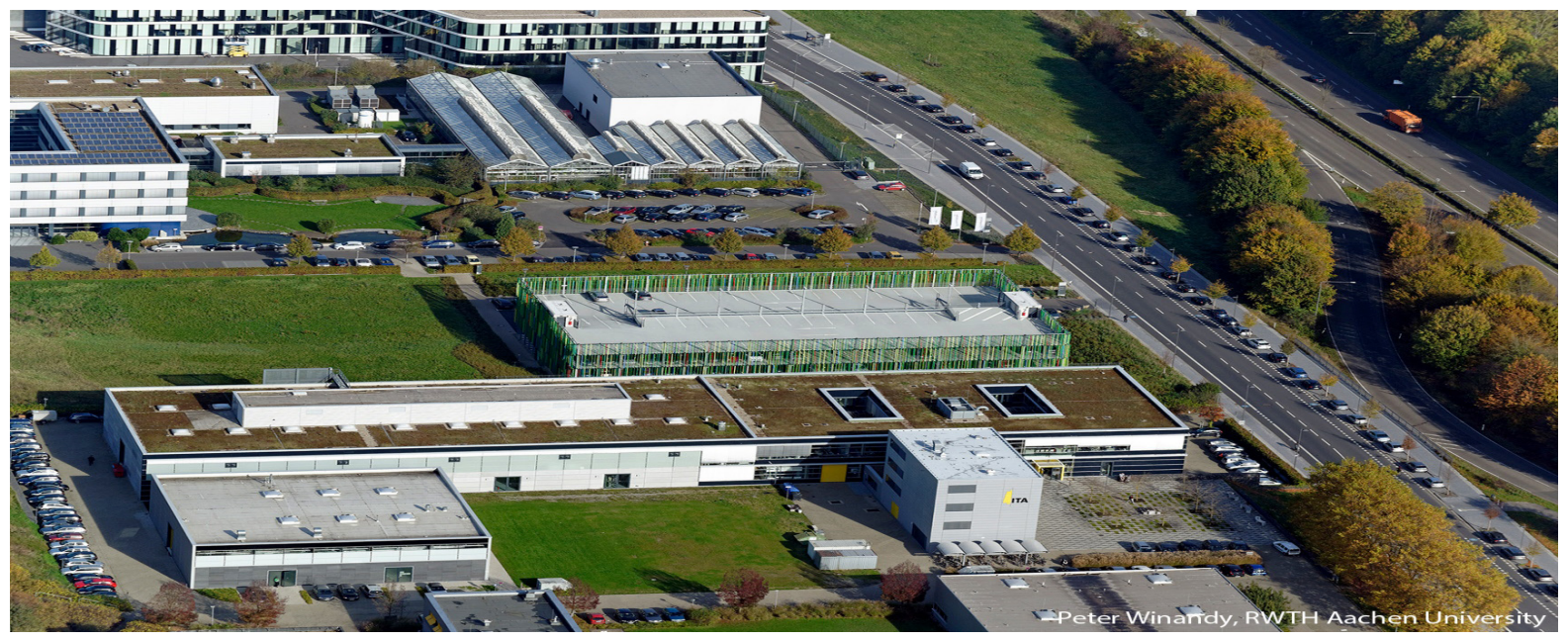

Figure I ITA location in Otto-Blumenthal-Straße on the Melaten Campus of RWTH Aachen (Picture: Jörg Stanzick)

With an interdisciplinary team of more than 50 scientists, technicians, assistants and students, the "Technical Fibres" department has been researching polymer fibres and their production for more than 50 years. Monofilament and multifilament technologies are studied and developed from laboratory to industrial scale, while the digitisation of the process steps is driven forward. The upstream processes including drying and compounding, as well as the downstream processes such as stretching and texturing are also covered in-house. Digitisation supports the quality monitoring of the processes and the institutes textile testing and polymer laboratories in the analysis of polymers and spun fibres.

All common thermoplastic synthetic fibre polymers are processed (a selection: PP, PE, PA, PET, PEEK and PEI) as well as compounded with a wide range of fillers and as blends. A special research focus lies on bio-based or biodegradable biopolymers (PLA, PHB, PBAT, PHA) and textile recycling. 

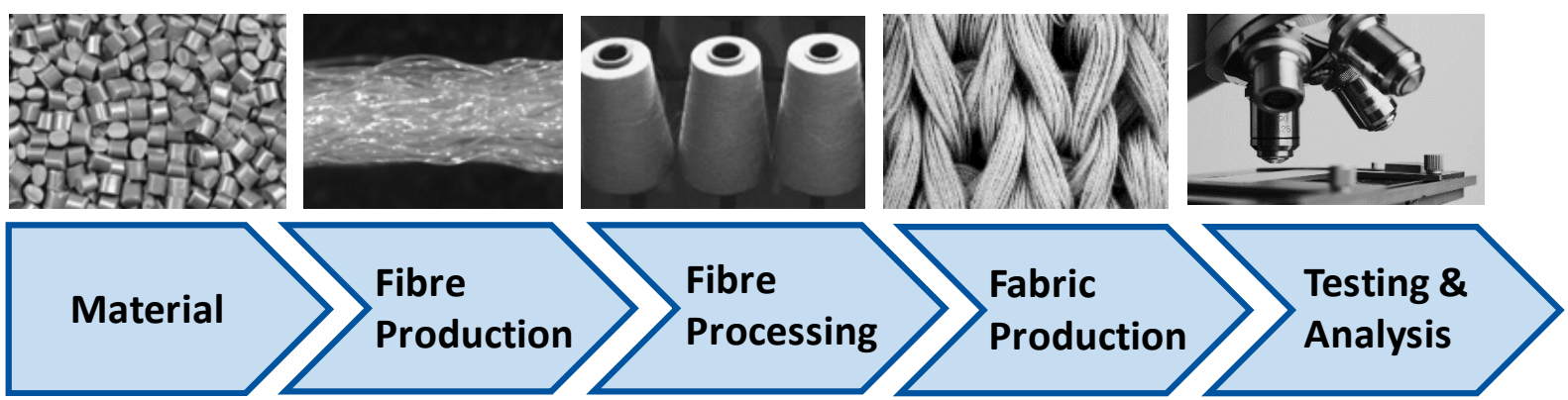

Figure $\mathbf{2}$ The textile process chain is completely covered at ITA. Several melt spinning plants and approx. 250 textile machines and test benches are available for this purpose.

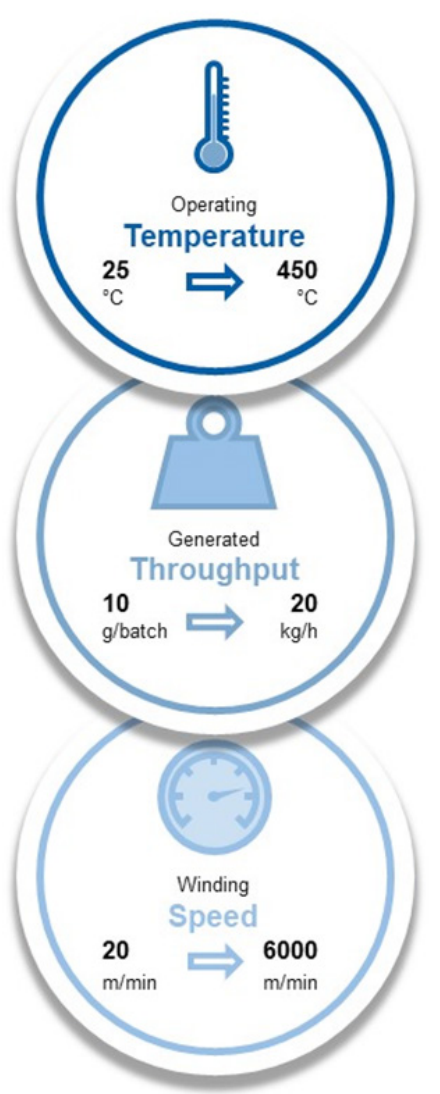

Figure 3 Possible process parametres.

A comprehensive testing and analysis technology supports the development processes during the melt spinning process as well as in the evaluation of the finished fibres and products. All common fibre and textile testing methods are available in the textile testing laboratory. In addition to optical measuring methods for fibre orientation and 3D deformation, high-speed and thermographic cameras, Laser Doppler Anemometry (LDA), Particle Image Velocimetry (PIV) as well as all common fibre, yarn and surface testing are carried out according to standard. A fully equipped polymer laboratory assists in the analysis of the raw materials. Measurement methods used are amongst others DSC-TGA, DMA, FT-IR, gas pycnometry, rheometry and Karl Fischer titration.

With a focus on future needs, the division is adressing the topics of energy, the environment, digitisation and recycling. Specific fields of research include functional fibres, active substance fibres, polymer optical fibres (POF) and conductive fibres, spinning process and structure creation, texturizing, digitisation and sustainability. In the following some current research projects of the individual topics are presented:

As part of a project in the field of functionalized fibres, fibres containing microcapsules are developed, which are capable of dissolving the fibre upon activation The idea of the project is to use these fibres as yarn for joining and subsequently for separating textile surfaces. The removal of oil contamination using functional textiles is the subject of other projects, as along with the development of flame retardants based on phosphorus-containing acrylates and methacrylates for the production of fire resistant aliphatic polyamide fibres (PA) (Figure 4)

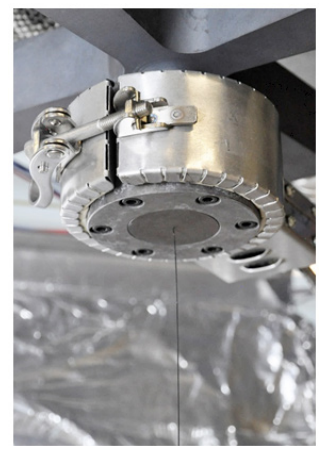

Figure 4 Monofilament nozzle of the ultrasonic dispersion unit.

Active ingredient fibres are spun with antibacterial properties or modified with scents for the use in pest control. In addition, components are being developed that improve the spinning process of particle-modified melts. For example, an ultrasonic dispersion unit has been realized, which allows an even distribution of particles in the filament.

Sensor technology based on optical or electrically conductive fibres enables continuous monitoring in a wide range of applications in areas such as aviation, automotive, energy, infrastructure, communication, construction, health and medicine. Innovative sensor fibres are likewise a main research area. Current projects include the use of polymer optical fibres for near-body sensors in magnetic resonance imaging and electrically conductive fibres integrated in composites for structural health monitoring. Further research in the field of polymer optical fibres includes the optimization of side light activation for 
illumination applications. Applications such as inductively heated fibres for cancer treatment are also being investigated in the field of conductive fibres (Figure 5).

Digitisation in the spinning process is being driven forward in various projects. Amongst others, a Smart Data Analytics platform is developed to increase product quality and the detection rate of defects. Furthermore, technology transfer concepts for linking textile process chains are created.

For the texturing of spun-dyed filament yarns, an innovative twisting unit is being developed, as well as abrasion-resistant yarn guides for the texturing lines.

In the sign of sustainability and the year of bio-economy 2020, the new space for innovation BioTex-Future was created. In a project with partners from various industries, bio-based textiles are produced here on the basis of sustainable raw materials. Biopolymers in the form of custom-made PHA polymers are currently developed in another project. As the winner of this year's university competition of the
German federal ministry of education and research, we will hold a workshop to clarify the effect of bio-economy and sustainability on the textile and fashion industry.

Industry-relevant fundamental research is currently performed in two Industry Research Groups (IRGs), who assemble a wide range of corporate partners. The research focus is determined by the industrial members. Technological, strategic or economic issues are considered exclusively for the partners. The IRG "Polymer Recycling" deals with the fibre-to-fibre recycling of textiles, while furthermore analysing the processability of the recycled polymers. The IRG "Meltspinning" deals with the mathematical modelling and simulation of the melt spinning process. The objective is to predict yarn properties on the basis of process parameters A model is created based on the systematic analysis of the material behaviour in the process, the resulting yarn properties, and the process parameters. Another IRG with the focus on monofilament technology is currently being established and will deal with topics such as electrically conductive and polymer optical fibres.

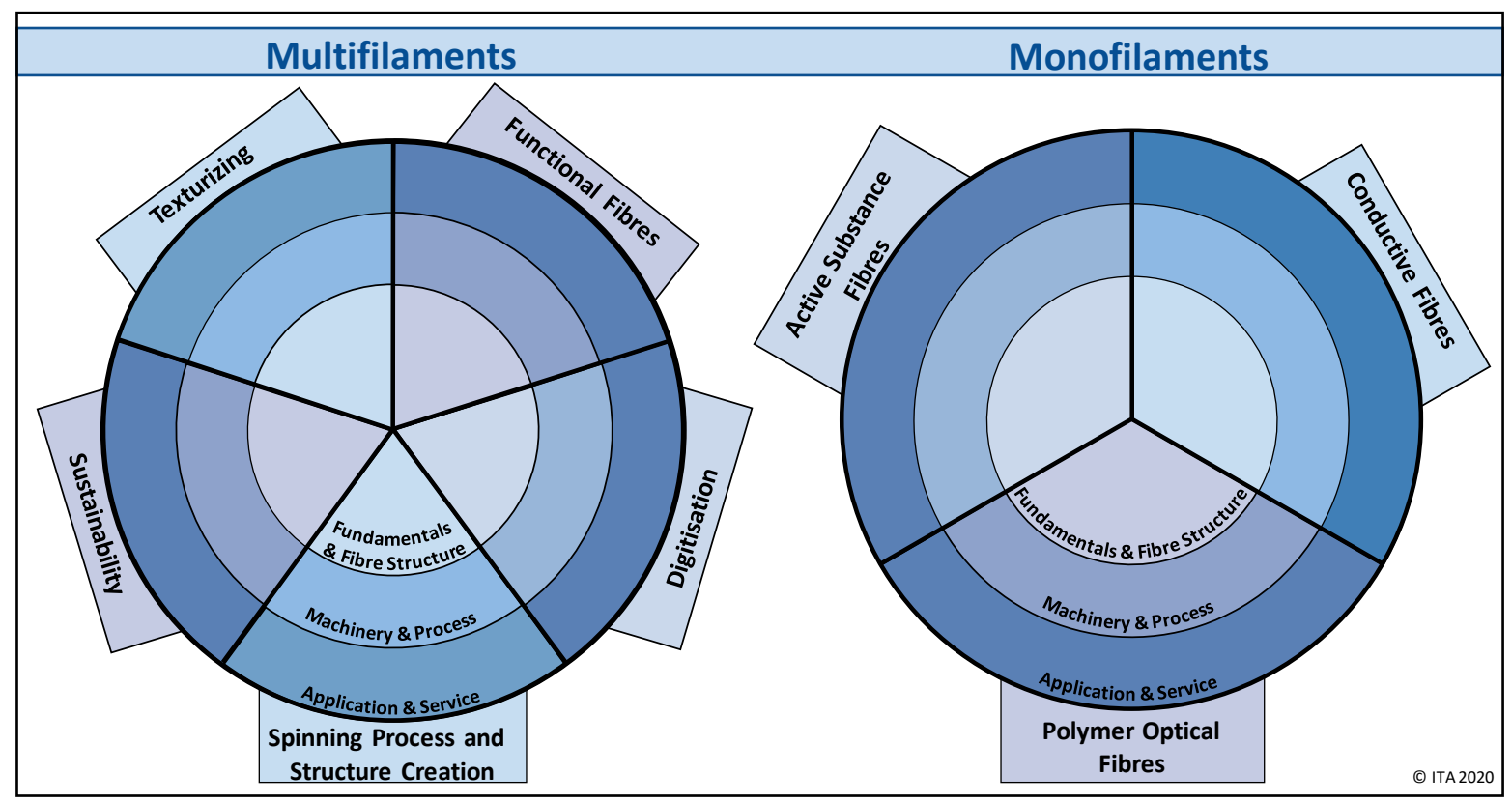

Figure 5 Research areas in the field of "Technical Fibres.

\section{Acknowledgments}

None.

\section{Funding}

None.

\section{Conflicts of interest}

No potential conflict of interest was reported by the authors. 